\title{
Attenuation Efficiency Of X-Ray And Comparison To Gamma Ray And Neutrons In Composite Metal Foams
}

\author{
Shuo Chen \\ Mohamed Bourham \\ Corresponding author: Afsaneh Rabiei ${ }^{\mathrm{a}}$ \\ ${ }^{a}$ Department of Mechanical and Aerospace Engineering, North Carolina State University, \\ Raleigh, NC 27695-7910, USA \\ Address: 3250 Engineering Bldg 3, 911 Oval Dr., Campus Box 7910, Raleigh, NC 27695-7910, \\ USA. \\ Tel.: +1 919-513-2674 \\ Fax: +1 919-515-7968 \\ Email: arabiei@ncsu.edu
}


Steel-steel composite metal foams (S-S CMFs) and Aluminum-steel composite metal foams (Al$\mathrm{S} C \mathrm{CMFs}$ ) with various sphere sizes and matrix materials were manufactured and investigated for nuclear and radiation environments applications. 316L stainless steel, high-speed T15 steel and aluminum materials were used as the matrix material together with 2, 4 and $5.2 \mathrm{~mm}$ steel hollow spheres to manufacture various types of composite metal foams (CMFs). High-speed T15 steel is selected due to its high tungsten and vanadium concentration (both high- $Z$ elements) to further improve the shielding efficiency of CMFs. This new type of S-S CMF is called High-Z steelsteel composite metal foam (HZ S-S CMF). Radiation shielding efficiency of all types of CMFs was explored for the attenuation of X-ray, gamma ray and neutron. The experimental results were compared with pure lead and Aluminum A356, and verified theoretically through XCOM and Monte Carlo Z-particle Transport Code (MCNP). It was observed that the radiation shielding effectiveness of CMFs is relatively independent of sphere sizes as long as the ratio of spherewall thickness to its outer-diameter stays constant. However, the smaller spheres seem to be more efficient in general due to the fine fluctuation in the gray value profile of their 2D MicroCT images. S-S CMFs and Al-S CMFs are respectively $275 \%$ and $145 \%$ more effective for Xray attenuation than Aluminum A356. Compared to pure lead, CMFs show adequate attenuation with additional advantages of being lightweight and more environmentally friendly. The mechanical performance of HZ S-S CMFs under quasi-static compression was compared to that of other classes of S-S CMF. It is observed that the addition of high-Z elements to the matrix of CMFs improved their shielding against X-rays, low energy gamma rays and neutrons, while maintained their low density, high mechanical properties and high-energy absorption capability.

\section{Introduction}

In recently years, the occurrence of severe accidents in Three Mile Island, Chernobyl, and Fukushima has attracted public's attention to develop advanced materials with improved radiation shielding properties, as well as excellent physical and mechanical performances to guarantee their safety, compliance, and efficiency in more aggressive environments surrounding nuclear reactors. Composite metal foams (CMFs) made of pre-fabricated metallic hollow spheres embedded in a metallic matrix have shown attractive attenuation efficiencies with lightweight and outstanding energy absorption properties (Chen et al., 2015, 2014a, 2014b; Neville and Rabiei, 2008; Vendra and Rabiei, 2007, 2010). Their unique structure and characteristics motivate creating another class of CMFs using a T15 high-speed steel powder as their matrix. This material is of particular interest due to its high- $Z$ contents of tungsten $(12.5 \mathrm{wt} \%)$ and vanadium (5.0 wt\%). Tungsten and vanadium, with atomic weights of 183.84 and 50.94, respectively, are effective shielding additives that would endow CMFs with improved shielding performance. Moreover, Tungsten offers high-temperature strength, good thermal conductivity, and compatibility for applications in nuclear environments (Leonard, 2012), whereas Vanadium has shown promising results in reducing radiation-induced damage (Bloom, 1998). The use of T15 high-speed steel as the matrix of CMFs may bring in enhanced radiation protection and mechanical properties.

The radiation from nuclear power plants and other radiation facilities is extremely varied in its character and ability to penetrate. The common forms of nuclear radiation are X-rays, gamma 
rays, and neutrons. In our previous attempt, CMFs have been investigated for attenuation of gamma rays and neutrons. For X-ray shielding, concrete is usually considered the most commonly used structural material. The major drawback of such shielding material is its high density, poor machinability and low mechanical stability. It has been reported that barium enriched concrete exhibits very good compressive strength and abrasion resistance in addition to possessing adequate shielding for X-rays comparable to pure lead (Nathuram, 2000). Similarly, barium based materials, such as barium silicate and barium aluminum silicates for their microstructure as self-reinforced composite (Yu et al., 2001), which also were used as additives in radiopaque fillers for making dental composite resin, and an enhancement of X-ray radiation shielding (Ghosh, 2012). These additional materials, which are responsible for enhancing X-ray shielding properties, are based on one or more of the compositions: barium aluminosilicate, iron phosphates, titanium, calcium, and magnesium. The chemical compositions of all these phases mainly consist of iron and aluminum. CMFs contain a fairly high quantity of iron $(65.7 \mathrm{w} \%$ in steel-steel composite metal foams (S-S CMFs) and $32.2 \mathrm{w} \%$ in aluminum-steel composite metal foams (Al-S CMFs)), and aluminum (47.3 wt\% in Al-S CMFs). It was therefore thought worthwhile to explore the feasibility of utilizing CMFs for X-ray shielding applications.

In this study, investigations on exploring the suitability of CMFs with various sphere sizes as Xray shields were carried out on a high-resolution microcomputed tomography (microCT) system, and the results were compared with those of Aluminum A356 and pure lead control samples to evaluate their shielding effectiveness. The benefit of introducing high-Z T15 powder to stainless steel matrix has been explored in terms of X-ray, gamma ray and neutron shielding. National Institute of Standards and Technology (NIST) XCOM code and Monte Carlo N-Particle Transport Code (MCNP) were used to verify the accuracy of the experimental data. Moreover, the mechanical performance of various classes of CMFs was verified by the means of quasistatic compressive testing.

\section{Materials, Processing and Sample Preparation}

Stainless steel hollow spheres (Hollomet GmbH, Dresden, Germany), Aluminum A356 casting alloy (TriAlCo, Inc), 316L stainless steel powder (North American Hoganas High Alloys LLC) and high-speed T15 steel powder (North American Hoganas High Alloys LLC) were used in processing the CMFs. The hollow spheres with outer diameters of 2, 4, and $5.2 \mathrm{~mm}$ were used for fabricating the CMFs. The compositional analysis of hollow spheres, aluminum A356 alloy, as well as 316L and high-speed T15 steel powders with sieve analysis are given in Table 1 and Table 2.

\subsection{Materials and Processing}

\subsubsection{Aluminum-steel composite metal foams}

Aluminum-steel composite metal foams (Al-S CMFs) consisting of hollow steel spheres and a solid aluminum A356 alloy matrix were processed through gravity casting technique. In the process of casting, the mold is assembled with the hollow spheres and preheated in a 3300 series high-temperature laboratory furnace (CM Furnaces, Inc., Bloomfield, NJ) to $700^{\circ} \mathrm{C}$ while the casting A356 is melted simultaneously in a clay graphite crucible. Molten Al is then cast and fills 
the spaces between the hollow spheres. More details of processing techniques are presented elsewhere (Rabiei and O’Neill, 2005; Vendra and Rabiei, 2007).

\subsection{2. $\quad$ Steel-steel composite metal foams}

Steel-steel composite metal foams (S-S CMFs) comprised of steel hollow spheres closely packed in either $316 \mathrm{~L}$ stainless steel powder or bimodal mixtures of $316 \mathrm{~L}$ and high-speed T15 steel powder were manufactured through powder metallurgy technique. Two permanent molds made of Grade 304 stainless steel were used: one with a rectangular cavity was used for manufacturing samples for radiation shielding studies, the dimension of the rectangular block were $51 \mathrm{~mm} \mathrm{x}$ $51 \mathrm{~mm} \times 89 \mathrm{~mm}$. The other one with a cylindrical cavity was used for quasi-static compression sample preparation, with $25.4 \mathrm{~mm}$ in diameter and $93.98 \mathrm{~mm}$ in height.

The mixture of hollow spheres and matrix powders were filled in the mold cavity, and vibrated to create a homogeneous and dense packing arrangement. The mixture was then isostatically pressed by hydraulic press (Dake 50H Model 907002) at 36MPa. The mold is then placed in a vacuum Hot Press (Centorr 600-4X6W4-26HP) for sintering. More details of manufacturing procedures of CMFs using powder metallurgy technique can be found elsewhere (Neville and Rabiei, 2008).

\subsubsection{High-Z steel-steel composite metal foam}

A bimodal mixture of 316L stainless steel powder and high-speed T15 steel powder was used as the matrix materials for manufacturing high-Z steel-steel composite metal foams (HZ S-S CMFs). The packing fraction $\phi_{\max }(\alpha, x)$ of the densest binary packing of spheres is a function of small to large particle radius ratio $\alpha=R_{S} / R_{L}$, and small particle relative concentration is defined as $x \equiv N_{S} /\left(N_{L}+N_{S}\right)$, with $R_{S}$ and $R_{L}$ the respective radius of the small and large particles, $N_{S}$ and $N_{L}$ the respective numbers of small and large spheres in the packing (Hopkins et al., 2011). In our study, the average particle radius of $316 \mathrm{~L}$ and T15 are calculated to be $R_{S}(316 L)=22.5 \mu m$ and $R_{S}(T 15)=43.0 \mu m$, respectively. According to the phase diagram in Ref. (Hopkins et al., 2011), at radius ratio $\alpha=0.5500$, the densest packing fraction $\phi_{\max }=$ 0.7793 can be reached by a hexagonal lattice system with two small spheres and one large sphere as in a periodic fundamental cell (Hopkins et al., 2011). Therefore, 316L and T15 powder were mixed in $22 \%$ to $78 \%$ volume ratio in a stainless steel vessel; the vessel was then placed on a ball mill (US Stoneware 1.8 Liter Ball Mill) for 30mins in order to achieve a uniform and homogeneous powder mixture.

All the processing steps of HZ S-S CMFs are the same as what is reported in previous study (Neville and Rabiei, 2008), except for decreasing the sintering temperature from $1200^{\circ} \mathrm{C}$ to $1180^{\circ} \mathrm{C}$. Since $1200^{\circ} \mathrm{C}$ is very close to the solidus temperature $\left(1215^{\circ} \mathrm{C}\right)$ of high-speed $\mathrm{T} 15$ steel powder (Bee et al., 1988). Therefore, a temperature below the solidus temperature of T15 powder $\left(1180^{\circ} \mathrm{C}\right)$ was chosen for $\mathrm{HZ} \mathrm{S-S} \mathrm{CMFs} \mathrm{manufacturing} \mathrm{to} \mathrm{prevent} \mathrm{any} \mathrm{liquid} \mathrm{phase}$ formation during sintering.

\subsection{Sample Preparation}




\subsubsection{X-ray transmission measurements samples}

Specimens used for X-ray transmission analysis are shown in Figure 1. Pure lead and Aluminum A356 alloy are classic forms of shielding materials for X-ray; therefore, they were selected as control samples in this study. Cold rolled pure lead (McMaster-Carr) with a thickness of 1/16" and thickness tolerance of $\pm 0.006 "$, contains a minimum of $98 \%$ pure lead content. A total of nine samples were prepared to evaluate their X-ray shielding capabilities:

- Al-S CMFs with $2 \mathrm{~mm}$ spheres [(2mm sphere) Al-S CMFs]

- Al-S CMFs with $4 \mathrm{~mm}$ spheres [(4mm sphere) Al-S CMFs]

- Al-S CMFs with 5.2mm spheres [(5.2mm sphere) Al-S CMFs]

- S-S CMFs with $2 \mathrm{~mm}$ spheres [(2mm sphere) S-S CMFs]

- S-S CMFs with $4 \mathrm{~mm}$ spheres [(4mm sphere) S-S CMFs]

- S-S CMFs with $5.2 \mathrm{~mm}$ spheres [(5.2mm sphere) S-S CMFs]

- High-Z S-S CMFs with $4 \mathrm{~mm}$ spheres [(4mm sphere) HZ S-S CMFs]

- Aluminum A356

- Pure lead

All samples were designed with the same cross sectional area (rectangular area $50.8 \mathrm{~mm} \mathrm{x}$ $89.3 \mathrm{~mm}$ ), and sample thickness (shown in Figure 1) is varied to provide areal density of $2 \mathrm{~g} / \mathrm{cm}^{2}$. This allowed direct comparisons of attenuation capabilities of different materials at a constant shield weight. Physical properties of CMFs, Aluminum A356 and pure lead are presented in Table 3.

\subsubsection{Gamma-ray and neutron transmission measurements samples}

The determination of gamma ray and neutron attenuation characteristics were carried out on eight sets of samples: Al-S CMFs and S-S CMFs, with either one of $2 \mathrm{~mm}, 4 \mathrm{~mm}$, and $5.2 \mathrm{~mm}$ spheres, HZ S-S CMFs with 4mm spheres, and control Aluminum A356 alloy. Aluminum A356 alloy is mainly selected as a reference material for shieling gamma and neutrons due to its low electric conductivity, high coefficient of thermal expansion and high corrosion resistance. In each set of samples, three different thicknesses were chosen to provide areal densities of 2,5 and $10 \mathrm{~g} / \mathrm{cm}^{2}$. Physical properties of CMFs, Aluminum A356 and pure lead are presented in Table 3.

\subsubsection{Quasi-static Compression Test Compression test sample preparation}

Two types of cylindrical samples, (4mm sphere) S-S CMFs and (4mm sphere) HZ S-S CMFs were prepared for quasi-static compression test. Each cylindrical sample $(25 \mathrm{~mm}$ in diameter) was cut into three smaller samples of $25 \mathrm{~mm}$ height giving a height/diameter ratio of 1 using a precision saw equipped with a diamond-wafering blade. Quasi static compression tests were performed using MTS 810 servo-hydraulic universal testing machine with a $980 \mathrm{kN}$ load cell and a crosshead speed of $1.25 \mathrm{~mm} / \mathrm{min}$. Tests were conducted at the Construction Facilities Laboratory (CFL) of NC State University. Each experiment was repeated at least three times and the average of three is reported. 


\section{Experimental Methods}

\subsection{X-ray Transmission Measurements}

A high-resolution microcomputed tomography (microCT) system (eXplore speCZT CT 120, TriFoil Imaging, Inc. Chatsworth, CA) was used to acquire X-ray projection images of CMFs, Aluminum A356 and pure lead. The X-ray source is a high-powered rotating-anode tube with the capability of running under variable energy and current at the focal spot size of $600 \mu \mathrm{m}$. In this study, the source was operated at maximum energy of $100 \mathrm{kVp}$, and a beam current of $50 \mathrm{~mA}$, exposure time of $20 \mathrm{~ms}$, and binning mode under $2 \times 2$. The CCD flat panel detector is comprised of 3500x2288 pixel elements covering an active area of $110 \times 75 \mathrm{~mm}^{2}$. The source and the detector positioning opposite to each other were set at a fixed distance of $450 \mathrm{~mm}$. During each measurement, the source and the detector move on a circular trajectory around a fixed carbon fiber tube sitting in the center. Both the experimental sample and the control materials, Aluminum A356 and pure lead, were placed inside the tube under the same field of view at each measurement. The data acquisition time was $2.5 \mathrm{sec}$ per projection and the images were formed from 360 views taken over a $360^{\circ}$ rotation. The Feldkamp's filtered back projection algorithm was used to reconstruct projection images with a resolution of $50 \mu \mathrm{m}$.

\subsection{Gamma-ray Transmission Measurements}

The gamma ray attenuation experiments were conducted in vertical narrow beam transmission geometry. Distance from gamma ray source to sample and from sample to detector is $256 \mathrm{~mm}$ and $327 \mathrm{~mm}$, respectively. The incident gamma radiation was provided using several gammaemitting sources: $3.0 \mathrm{mCi}{ }^{60} \mathrm{Co}$ (photon energies of $1.332 \mathrm{MeV}$ and $1.173 \mathrm{MeV}$ ), $1.8 \mathrm{mCi}{ }^{137} \mathrm{C}_{\mathrm{S}}$ (photon energy of $0.662 \mathrm{MeV}$ ), $13.5 \mathrm{mCi}{ }^{124} \mathrm{Am}$ (photon energy of $0.060 \mathrm{MeV}$ ), and $5.0 \mathrm{mCi}{ }^{133} \mathrm{Ba}$ (photon energies of $0.356 \mathrm{MeV}$ and $0.081 \mathrm{MeV}$ ). Both incident $\left(I_{0}\right)$ and transmitted $(I)$ beam intensities were recorded at a fixed count of 200,000. Three readings were measured at each energy level, and the average of the three was used for mass attenuation coefficient calculation. Theoretical values were obtained through National Institute of Standards and Technology (NIST) XCOM database which was developed by Berger and Hubbell (Berger and Hubbell, 2010). The results have been compared with experimental data for validation. More detailed can be found elsewhere (Chen et al., 2014a, 2014b).

\subsection{Neutron Transmission Measurements}

The evaluation of neutron transmission measurements were carried out at the Neutron Powder Diffractometer beam facility at North Carolina State University 1.0MW PULSTAR reactor with $0.64 \times 10^{5} \mathrm{n} / \mathrm{cm}^{2} / \mathrm{s}$ beam intensity at the guide aperture. The sample was placed at $36 \mathrm{~cm}$ downstream from the neutron source and $22 \mathrm{~cm}$ upstream from the detector. For each sample, the background $\left(\Phi_{b}\right)$ and transmitted $\left(\Phi_{t}\right)$ neutron intensities were measured in a 600s interval, which was reasonable enough to obtain a statistical uncertainty in the range of $0.21 \%$ to $0.62 \%$; the neutron transmission was calculated using $T=\left(\Phi_{t}-\Phi_{b}\right) /\left(\Phi_{0}-\Phi_{b}\right)$ after acquiring the corresponding open beam $\left(\Phi_{o}\right)$ at a constant time of 10s. Theoretical values of the neutron transmission were calculated by Monte Carlo N-Particle (MCNP5) (MCNP, 1986) developed at 
Los Alamos National Laboratory. More detailed can be found elsewhere (Chen et al., 2015, 2014b).

\subsection{Quasi-static Compression Test}

Quasi-static compression tests were conducted using an MTS 810 universal testing machine with a $980 \mathrm{kN}$ load cell and a crosshead speed of $1.25 \mathrm{~mm} / \mathrm{min}$ (Neville and Rabiei, 2008) at Construction Facilities Laboratory (CFL) at North Carolina State University. A digital camera (Canon, EOS 7D) was employed to observe the samples' deformation under compression loading. Vacuum grease was applied to both ends of each testing samples in order to lubricate the contact surfaces between the samples and the testing machine to minimize friction and resulted barreling effects. Each measurement was repeated three times. Load capacity and compression were imported into computer by sensor, and collected data were treated to obtained stress-strain curve.

\subsection{Characterization}

Open source software Image $\mathrm{J}$ version 1.49s (Image $J$, 2015) was used for image processing in order to achieve good quality images for quantitative analysis. It also enables to analyze the general characteristics of the materials by displaying the image histogram or plotting the profile of intensity values. Grey levels represent number of photons that were able to penetrate through specimen along the path of the X-ray beam.

\section{Results and Discussions}

\subsection{X-ray transmission results}

Figure 2 shows projection images of CMFs in comparison with two reference materials: Aluminum A356 and pure lead. As can be seen, the porous media within the matrix is uniform, homogeneous and isotropic, which provide CMFs excellent consistency and stability in engineering design applications. In addition, these images open up an opportunity to directly visualize complex 3D pore structures into simplified 2D description and allow for more detailed correlations to be calculated. As can be seen from each image, both S-S CMFs and Al-S CMFs offer more X-ray shielding efficiency than that of Aluminum A356, and less attenuation than that of pure lead. At a given sphere size, S-S CMFs (darker in the image) seems to be more absorbing comparing to Al-S CMFs, and improved attenuation efficiency of (4mm sphere) HZ S-S CMFs as compared to (4mm sphere) S-S CMFs can be observed as a result of the existence of tungsten $(12.5 \mathrm{wt} \%)$ and vanadium $(5.0 \mathrm{wt} \%)$ in the matrix (Table 2). Projection images can provide not only geometrical information, but also numerical insights of each sample by drawing a vertical line from the top to the bottom (shown in Figure $3(\mathrm{~g})$ ) in each image and analyze it by image $\mathrm{J}$ program.

Figure 3 presents the corresponding vertical line profile from the top to the bottom in each projection image in Figure 2. Grey value indicates the total number of pixels corresponding to the transmitted photon intensity level. High peaks with more grey values indicate more photon transmission, whereas low peaks show less photon transmission. The gaps in between CMFs and 

which were shown as two narrow sharp peaks at the distance of $50 \mathrm{~cm}$ and $60 \mathrm{~cm}$ (Figure $3 \mathrm{~g}$ ). The profile line in the range $0-50 \mathrm{~cm}$ indicate porous structure in CMFs samples, $(5.2 \mathrm{~mm}$ sphere) CMFs are more wavy compared to $(2 \mathrm{~mm}$ sphere) CMFs, resulted from the relatively lager hollow sphere size. In order to quantitatively measure shielding effectiveness of samples, the mean pixel count was calculated by using Image J program and presented in Figure 4.

Figure 4 shows the mean grey value of all X-ray images taken from each one of the samples. As can be seen, for a given areal density of shielding material $\left(2 \mathrm{~g} / \mathrm{cm}^{2}\right)$, the effect of sphere sizes on the shielding effectiveness of both Al-S and S-S CMFs is insignificant. This can be due to the fact that the sphere wall thickness $(t)$ to sphere radius $(r)$ ratio is the same in all samples and as such, the amount of shielding material in all samples is the same, regardless of their sphere sizes. However, the smaller spheres seem to be more efficient in general due to the fine fluctuation in the gray value profile of their 2D Micro-CT images. It was calculated that Al-S CMFs and S-S CMFs are respectively $145 \%$ and $275 \%$ more effective in shielding X-rays compared to Aluminum A356. This is attributed to higher contents of iron in the sphere wall and within S-S CMFs matrix. This shielding efficiency can be further enhanced to $398 \%$ in HZ S-S CMFs as a result of additional elements of tungsten $(12.5 \mathrm{wt} \%)$ and vanadium $(5.0 \mathrm{wt} \%)$ in the T15 matrix. Even though pure lead is still the predominant shield material for X-ray, its toxic nature to human organ and tissues has largely limited its applications. It is worth noting that, in Figure 5, HZ S-S CMFs have an average density of $2.69 \mathrm{~g} / \mathrm{cm}^{3}$, and it is found to be $25 \%$ more absorbing than regular S-S CMFs with an average density of $2.73 \mathrm{~g} / \mathrm{cm}^{3}$. This encouraging result is a breakthrough in shielding X-rays and opens the door for future work on further improving the shielding capability of CMFs while maintaining the advantages of its unique low-weight and outstanding physical and mechanical characteristics.

In table 4, the mass attenuation coefficient was computed using experimentally measured X-ray transmission and theoretically obtained mass attenuation coefficient values using XCOM. As can be seen the experimental and theoretical data are in good agreement. The over-all uncertainty could be determined by errors in areal density measurements and counting statistic, as well as non-homogeneous porous structure of samples and the resulting multiple scattering.

\subsection{Gamma ray transmission results}

\subsection{1. $\quad$ Effect of sample thickness on gamma ray shielding}

Gamma ray transmission data on (4mm sphere) CMFs reported in our previous work (Chen et al., 2014a) are reported in this section for comparison with the new data collected from the $(2 \mathrm{~mm}$ sphere) and (5.2mm sphere) CMFs, as well as (4mm sphere) HZ S-S CMFs. Figure 6 presents measured transmission gamma ray for all samples at six different photon energies. It is apparent that the general trend is a decrease with decreasing of the incident energy. In each plot, the transmission profile decreases with increasing of sample thickness. The relation can explain the strong dependency of transmission on the sample thickness is: $I=I_{0} \exp (-u x)$, where $u\left(\mathrm{~cm}^{-1}\right)$ is the linear attenuation coefficient and $x(\mathrm{~cm})$ is the sample thickness. As can be seen from the equation, transmission decreases exponentially with increasing of the sample thickness. 
At higher energy level $(1.332 \mathrm{MeV}, 1.173 \mathrm{MeV}$ and $0.662 \mathrm{MeV})$, the transmission curves are overlapping. This is due to the fact that the effective interaction mechanism between gamma ray and matter in energy ranges from $0.5 \mathrm{MeV}$ to $10 \mathrm{MeV}$ is Compton scattering. Compton scattering involves the scattering of photons by charged particles where both energy and momentum are transferred to the charged particle, while the photon moves off with a reduced energy and a change of momentum (Hütt et al., 2000). In this interaction, Compton scattering causes increasing function of electron density of the material. All elements contain approximately the same number of electrons per unit mass. Hence, the displayed transmission profile has almost the same shape under ${ }^{60} \mathrm{Co}$ and ${ }^{137} \mathrm{Cs}$ sources.

When energy drops to $0.356 \mathrm{MeV}$, photoelectric absorption $(\alpha)$ starts to take place. The difference between Al-S CMFs and S-S CMFs in shielding gamma rays in such energy levels is distinguishable in Figure 6(d). The photoelectric absorption process converts electromagnetic energy of a gamma ray photon into kinetic energy of a charged particle (Knoll, 2000). At a given photon energy, the interaction is proportional to the effective atomic number $\left(Z_{\text {eff }}\right)$ by the relation $\left(\alpha \propto Z_{\text {eff }}^{5} / E^{7 / 2}\right)$ (Jevremovic, 2009). As can be seen in Figure 6(e), a clear difference between Al-S CMF, S-S CMF and Aluminum A356 for attenuating gamma rays can be observed. This is mainly due to the fact that higher effective atomic number $Z_{\text {eff }}$ can offer better attenuation efficiency. As given in Table 5, the average values of $Z_{\text {eff }}(0.08 \mathrm{MeV})$ for HZ S-S CMF, S-S CMF, Al-S CMF and Aluminum A356 are calculated to be 29.5, 26.5, 22.4, and 13.3, respectively, and these data are in agreement with the transmission curves shown in Figure 6(e). With further decreasing of the photon energy to $0.060 \mathrm{MeV}$ (Figure 6(f)), the incident gamma ray flux was fully shielded by CMFs at areal density of $5 \mathrm{~g} / \mathrm{cm}^{2}$. The specimen with greater $Z_{\text {eff }}$ (shown in table 5) shows better attenuation efficiency.

\subsection{3. $\quad$ Effect of sphere size on gamma ray shielding}

For a given areal density of shielding, the difference between sphere sizes on both Al-S and S-S CMFs is insignificant as long as the ratio of sphere-wall thickness (t) to its outer-diameter (R) stays constant, that is to say, the decrease in gamma ray attenuation by the air inside the spheres is balanced out by the increase in gamma ray attenuation by the sphere wall under the same $t / R$ ratio. However, the smaller spheres seem to be more efficient in general due to the fine fluctuation and homogeneity in the gray value profile shown in their 2D Micro-CT images.

The mass attenuation coefficient obtained for all specimens at six different photon energies are presented in Table 6, and compared with the theoretical results computed by XCOM code. The error involved in over all experimental values is lying between 2-3\% with the theoretical values. As shown in Figure 7, the determined mass attenuation coefficients fall onto the theoretical curve and show a good agreement within the experimental error.

\subsection{Neutron transmission results}


In order to compare the neutron shielding efficiency of our new HZ S-S CMF with that of other traditional CMFs, the neutron transmission measurement result for other CMFs are retrieved from our previous work (Chen et al., 2015) and are plotted in the same Figure. As shown in Figure 8, it is obvious that $\mathrm{HZ}$ matrix enhances the neutron attenuation of the foams considerably. The (4mm sphere) HZ S-S CMF was found to be respectively, 60\%, 100\%, and $180 \%$ more effective than those of S-S CMF, Al-S CMF, and Aluminum A356. It is well known that a large neutron cross-section is the key criterion for better neutron attenuation capability. The significant improvement of HZ S-S CMF in terms of neutron shielding is attributed to the presence of high- $Z$ elements such as tungsten and vanadium in the matrix materials. The neutron cross-section of tungsten and vanadium are respectively $18.4 \mathrm{~b}$ and $5.06 \mathrm{~b}$. It should be noted that tungsten and vanadium are identified as low activation elements (Bloom, 1998), which can offer an additional advantage of radiation damage resistance to $\mathrm{HZ} \mathrm{S-S} \mathrm{CMF.} \mathrm{Moreover,} \mathrm{it} \mathrm{is}$ anticipated that the presence of pre-existing partial deboning between spheres and matrix, the air trapped inside the spheres as well as micro-porosity inside sphere walls and the matrix could reduce volumetric swelling in case of exposure to high-flux neutron. These unique properties maximized the attractiveness of CMFs in general and new class of HZ S-S CMF in particular for nuclear structural applications from the viewpoints of safety and stability.

A 3D model was built by using MCNP5 code to evaluate neutron interaction with CMFs. The hollow sphere packing density in CMFs was reported to be 0.59 (Rabiei and Vendra, 2009), which is classified as a random loose packing. The real geometry of CMFs is not easy to characterize due to the random loose packing of the spheres in the matrix. Therefore, geometric idealization was employed in the modeling by utilizing periodic unit cell. As shown in Figure 9, HZ S-S CMF was proposed through three sphere arrangements: (a) simple cubic (SC) structure (b) body center cubic (BCC) structure, and (c) face center cubic (FCC) structure with packing factor of $0.52,0.68$, and 0.74 , respectively. The simulated transmission curves with respect to the thickness of HZ S-S CMF are given in Figure 10. It was expected that the overall performance of the shielding structure, in terms of neutron attenuation capability, would be highly dependent on the sphere arrangement within the matrix. The SC structure exhibits the highest attenuation efficiency. This is attributed to its lowest sphere packing density, which is a reflection of lowest percentage of air content in the structure. The lower the packing factor, the less the transmission as a result of the less content of air. It can be clearly seen in Figure 10 that experimental data falls in between SC and BCC theoretical curves, and it is in a good agreement with the model prediction.

\subsection{Compression test results}

The compressive stress-strain relationships of (4mm sphere) S-S CMF and (4mm sphere) HZ S-S CMF subjected to quasi-static loading are shown in Figure 11. It can be seen that these two CMFs have similar stress-strain behavior that includes three distinct regions: (I) a linear elastic region appears at a low strain, (II) A plateau region covers up to over $50 \%$ strain and (III) a densification region after the plateau region. During the plateau region all the spheres and porosities in the material are gradually collapse through a large irreversible plastic deformation along with a slight increase in stress value. However, the stress increases drastically with slight increase of strain in densification region. Both (4mm sphere) S-S CMF and (4mm sphere) HZ S$S$ continue a uniform deformation from the early stage of the compression to the higher strains, 
no evidence of visible collapse bands were observed in specimen. The material maintains its integrity and uniform deformation throughout the entire compression process. A slightly decrease in the plateau stress, from $127 \mathrm{MPa}$ of (4mm sphere) S-S CMF to $114 \mathrm{MPa}$ of (4mm sphere) HZ S-S CMF, can be observed. This is resulted from the decrease of the specimen density from $3.2 \mathrm{~g} / \mathrm{cm}^{3}$ ((4mm sphere) S-S CMFs) to $2.9 \mathrm{~g} / \mathrm{cm}^{3}$ ((4mm sphere) HZ S-S CMF). The density decrease might be linked with the slightly lower sintering temperature of (4mm sphere) HZ S-S CMF (Figure 12a, indicated in red arrows), which could have caused the remaining of some un-sintered particles in the material. As a result, some irregular micro-porosities appeared within the matrix of HZ S-S CMF. Whereas in (4mm sphere) S-S CMF (Figure 12b), matrix materials are very well sintered, no micro-porosities occurred. The results of this work indicate that further investigations are needed to gain more insight into the importance of matrix composition, sintering temperature and time, and their quantitative contribution to the stress response.

\section{Conclusions}

X-ray microcomputed tomography is a powerful and effective tool to supply significant qualitative and quantitative information on attenuation effectiveness of pore-scale materials. In this work, we tested nine samples with such system. Comparing CMFs with Aluminum A356, Al-S CMFs and S-S CMFs were observed to be respectively $145 \%$ and $275 \%$ more effective than Aluminum A356 in terms of X-ray attenuation. As compared with pure lead, CMFs show adequate shielding with additional advantages of being environmentally friendly, heat rejection and energy absorption capabilities. Introducing the T15 powder into the S-S CMF matrix materials improved the shielding efficiency for all three types of radiation, X-ray, gamma ray and neutrons with additional advantages of low density and high-energy absorption capabilities. Therefore, CMFs can preferably be used for the construction of X-ray diagnostic rooms, CTscanner rooms, nuclear power plants and other nuclear facilities to provide adequate shielding with favorable heat rejection, energy absorption, and being more environmentally friendly. This study establishes an opportunity and opens a room for future modifications of matrix materials or sphere wall compositions of CMFs with improved shielding performance while maintaining its excellent mechanical properties in high radiation environment in nuclear structures.

\section{Acknowledgments}

This research is performed using funding received from the DOE Office of Nuclear Energy's Nuclear Energy University Programs \#CFP-11-1643. Thanks to the UNC Biomedical Research Imaging Center (BRIC) Small Animal Imaging facility for providing microCT imaging service, and the imaging facility support from NCI center grant P30-CA016086-35-37.

\section{References}

Bee, J.V., Brewin, P.R., Nurthen, P.D., Wood, J.V., 1988. Sintering mechanisms in vacuum sintered M2 and T15 high speed steel powders. Met. Powder Rep. 43, 177-184.

Berger, M.J., Hubbell, J.H., 2010. XCOM: Photon Cross Sections Database. National Institute of Standards and Technology, Gaithersburg, MD. 
Bloom, E.E., 1998. The challenge of developing structural materials for fusion power systems. J. Nucl. Mater. 258-263, Part 1, 7-17. doi:10.1016/S0022-3115(98)00352-3

Chen, S., Bourham, M., Rabiei, A., 2015. Neutrons attenuation on composite metal foams and hybrid open-cell Al foam. Radiat. Phys. Chem. 109, $27-39$.

Chen, S., Bourham, M., Rabiei, A., 2014a. Novel light-weight materials for shielding gamma ray. Radiat. Phys. Chem. 96, 27-37.

Chen, S., Bourham, M., Rabiei, A., 2014b. Applications of Open-cell and Closed-cell Metal Foams for Radiation Shielding. Procedia Mater. Sci., 8th International Conference on Porous Metals and Metallic Foams 4, 293-298.

Ghosh, N.N., 2012. Development of Aqueous Sol-Gel Method for Synthesis of Nanostructured SiO2-BaO Powder for Use in Dental Composite Resins, in: Laine, R.M., Hu, M., Lu, S. (Eds.), Ceramic Nanomaterials and Nanotechnologies IV. John Wiley \& Sons, Inc., Hoboken, NJ, USA, pp. 207-212.

Hopkins, A.B., Stillinger, F.H., Torquato, S., 2011. Densest binary sphere packings. ArXiv11114917 Cond-Mat.

Hütt, M.-T., L'vov, A.I., Milstein, A.I., Schumacher, M., 2000. Compton scattering by nuclei. Phys. Rep. 323, 457-594. doi:10.1016/S0370-1573(99)00041-1

Image J, 2015. . National Institutes of Health.

Jevremovic, T., 2009. Nuclear Principles in Engineering. Springer US, Boston, MA.

Knoll, G.F., 2000. Radiation Detection and Measurement, third ed. John Wiley and Sons, New Jersey. (Chapter 2), third edition. ed. John Wiley and Sons, New Jersey.

Leonard, K.J., 2012. 4.06 - Radiation Effects in Refractory Metals and Alloys, in: Konings, R.J.M. (Ed.), Comprehensive Nuclear Materials. Elsevier, Oxford, pp. 181-213.

MCNP, 1986. MCNP-A general Monte Carlo code for neutron and photon transport. Los Alamos National Laboratory, Los Alamos, New Mexico, Report LA-7396-M, Rev. 2.

Nathuram, R., 2000. Photon attenuation characteristics of radiation shielding materials.

Neville, B.P., Rabiei, A., 2008. Composite metal foams processed through powder metallurgy. Mater. Des. 29, 388-396.

Rabiei, A., O'Neill, A.T., 2005. A study on processing of a composite metal foam via casting. Mater. Sci. Eng. A 404, 159-164.

Rabiei, A., Vendra, L.J., 2009. A comparison of composite metal foam's properties and other comparable metal foams. Mater. Lett. 63, 533-536.

Vendra, L.J., Rabiei, A., 2007. A study on aluminum-steel composite metal foam processed by casting. Mater. Sci. Eng. A 465, 59-67.

Vendra, L., Rabiei, A., 2010. Evaluation of modulus of elasticity of composite metal foams by experimental and numerical techniques. Mater. Sci. Eng. A 527, 1784-1790.

Yu, F., Nagarajan, N., Fang, Y., White, K.W., 2001. Microstructural Control of a 70\% Silicon Nitride- 30\% Barium Aluminum Silicate Self-Reinforced Composite. J. Am. Ceram. Soc. 84, 13-22. doi:10.1111/j.1151-2916.2001.tb00601.x 
Figure 1: Digital images of CMFs produced with $2 \mathrm{~mm}, 4 \mathrm{~mm}$, and $5.2 \mathrm{~mm}$ spheres, along with control samples - Aluminum A356 and pure lead, at areal density of $2 \mathrm{~g} / \mathrm{cm}^{2}$

Figure 2: Micro-CT 2D projection images of (a) (2mm sphere) Al-S CMF, (b) (4mm sphere) Al$\mathrm{S} \mathrm{CMF}$, (c) (5.2mm sphere) Al-S CMF, (d) (2mm sphere) S-S CMF, (e) (4mm sphere) S-S CMF, (f) (5.2mm sphere) S-S CMF, and (g) (4mm sphere) HZ S-S CMF,

Figure 3: Gray value profile from top to bottom in each projection image of the 2D Micro-CT images using an arbitrary vertical line indicated as a yellow line in Figure 2(g)

Figure 4: Histogram of mean gray values associated with the projection images of the 2D MicroCT of CMFs, Aluminum A356 and pure lead

Figure 5: Histogram of mean gray values associated with the projection images of the 2D MicroCT of pure lead, (4mm sphere) S-S CMF, (4mm sphere) HZ S-S CMFs

Figure 6: Gamma-ray transmission as a function of thickness for all specimens at six different photon energies (a) ${ }^{60} \mathrm{Co}(1.332 \mathrm{MeV})$, (b) ${ }^{60} \mathrm{Co}(1.173 \mathrm{MeV}),(\mathrm{c}){ }^{137} \mathrm{Cs}(0.662 \mathrm{MeV}),(\mathrm{d}){ }^{133} \mathrm{Ba}$ $(0.356 \mathrm{MeV}),(\mathrm{e}){ }^{133} \mathrm{Ba}\left(0.081 \mathrm{MeV}\right.$, and (f) ${ }^{241} \mathrm{Am}(0.060 \mathrm{MeV})$

Figure 7: Comparison of experimental and theoretical mass attenuation coefficients of (a) (2mm sphere) CMFs and Aluminum A356, (b) (4mm sphere) CMFs and Aluminum A356, (c) (5.2mm sphere) CMFs and Aluminum A356, (a'), (b'), and (c') are respectively zoomed in images from boxed area of (a), (b), and (c)

Figure 8: Neutron transmission as a function of samples thickness

Figure 9: Model geometries of different arrangements for (4mm sphere) HZ S-S CMFs using MCNP code (a) simple cubic structure (b) body center cubic structure and (c) face center cubic structure

Figure 10: Comparison of neutron experimental and theoretical transmission results for (4mm sphere) HZ S-S CMFs

Figure 11: Stress-strain curves of (4mm sphere) S-S CMF and (4mm sphere) HZ S-S CMF

Figure 12: Digital images showing the cross-section of (a) (4mm sphere) HZ S-S CMF, and (b) (4mm sphere) S-S CMF before quasi-static testing. Red arrows indicate the micro-porosity in the matrix of (4mm) HZ S-S CMF 


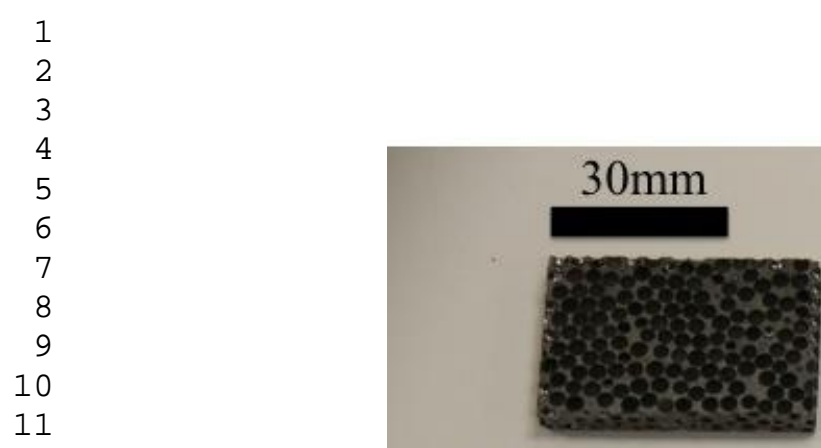

12

13

14

15

16

17

18

19

20

21

22

23

24

25

26

27

28

29

30

31

32

33

34

35

36

37

38

39

40

41

42

43

44

45

46

47

48

49

50

51

52

53

54

55

56

57

58

59

60

61

62

63

64

65
$T=$ Thickness of the sample

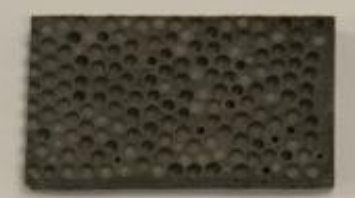

(5.2 $\mathrm{mm}$ sphere) S-S CMF $\mathrm{T}=7.9 \mathrm{~mm}$

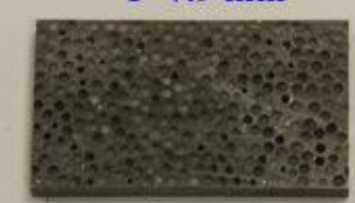

(4mm sphere) Al-S CMF

(4mm sphere) S-S CMF $\mathrm{T}=7.2 \mathrm{~mm}$

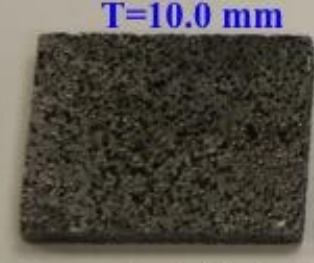

(2mm sphere) Al-S CMF $\mathrm{T}=\mathbf{1 0 . 8} \mathrm{mm}$

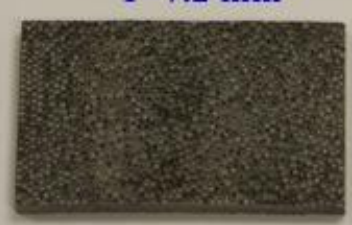

(2mm sphere) S-S CMF $\mathrm{T}=7.7 \mathrm{~mm}$

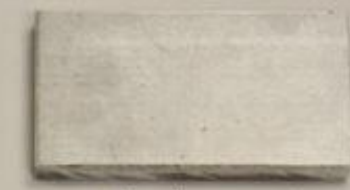

Aluminum A356

$\mathrm{T}=7.4 \mathrm{~mm}$

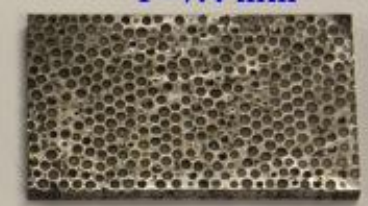

(4mm sphere) HZ S-S CMF $\mathrm{T}=7.7 \mathrm{~mm}$

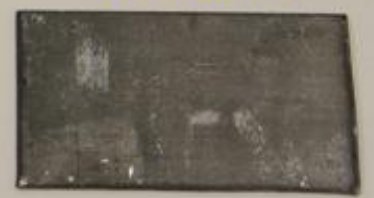

Pure lead $\mathrm{T}=1.8 \mathrm{~mm}$ 


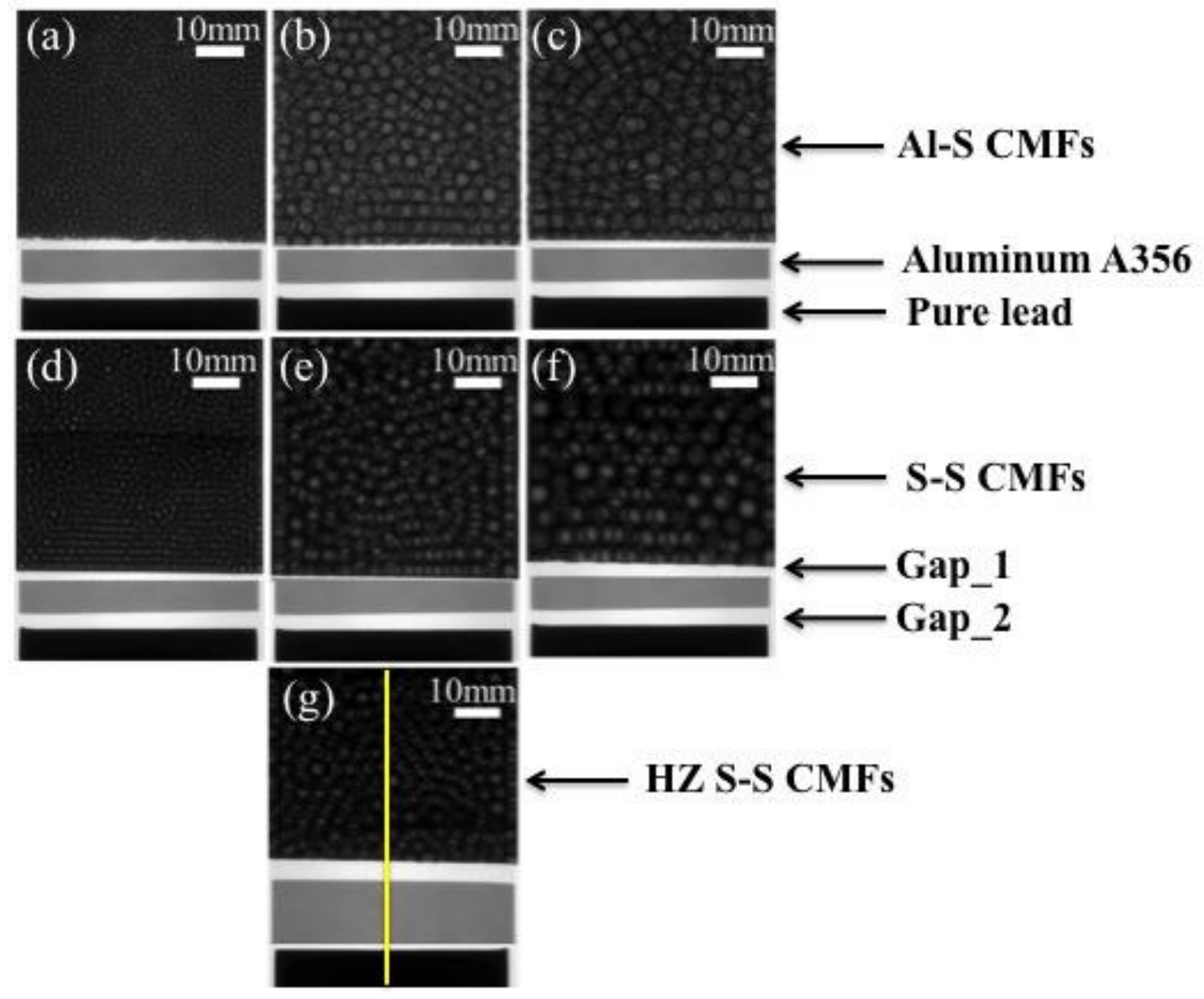

38

39

40

41

42

43

44

45

46

47

48

49

50

51

52

53

54

55

56

57

58

59

60

61

62 

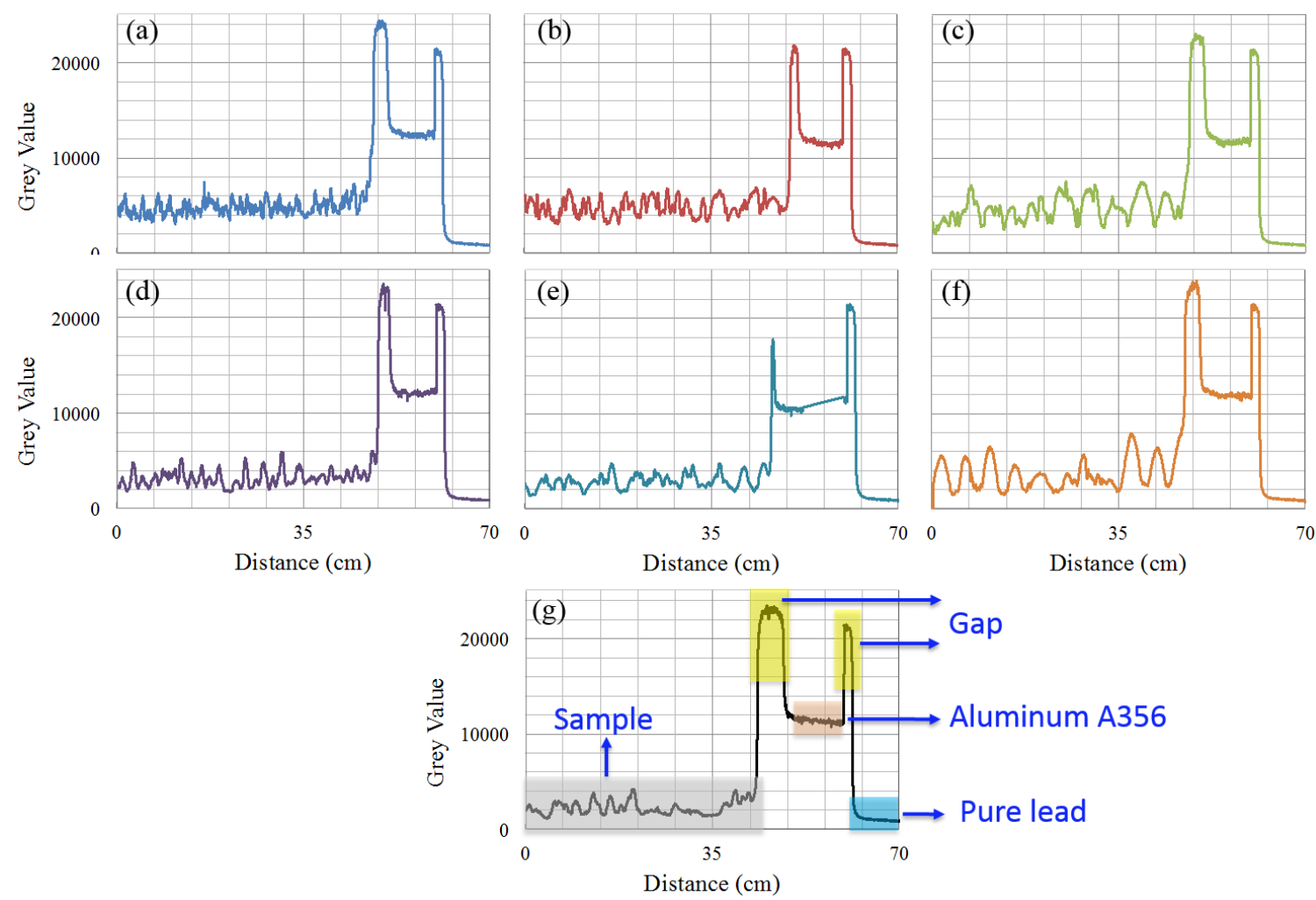


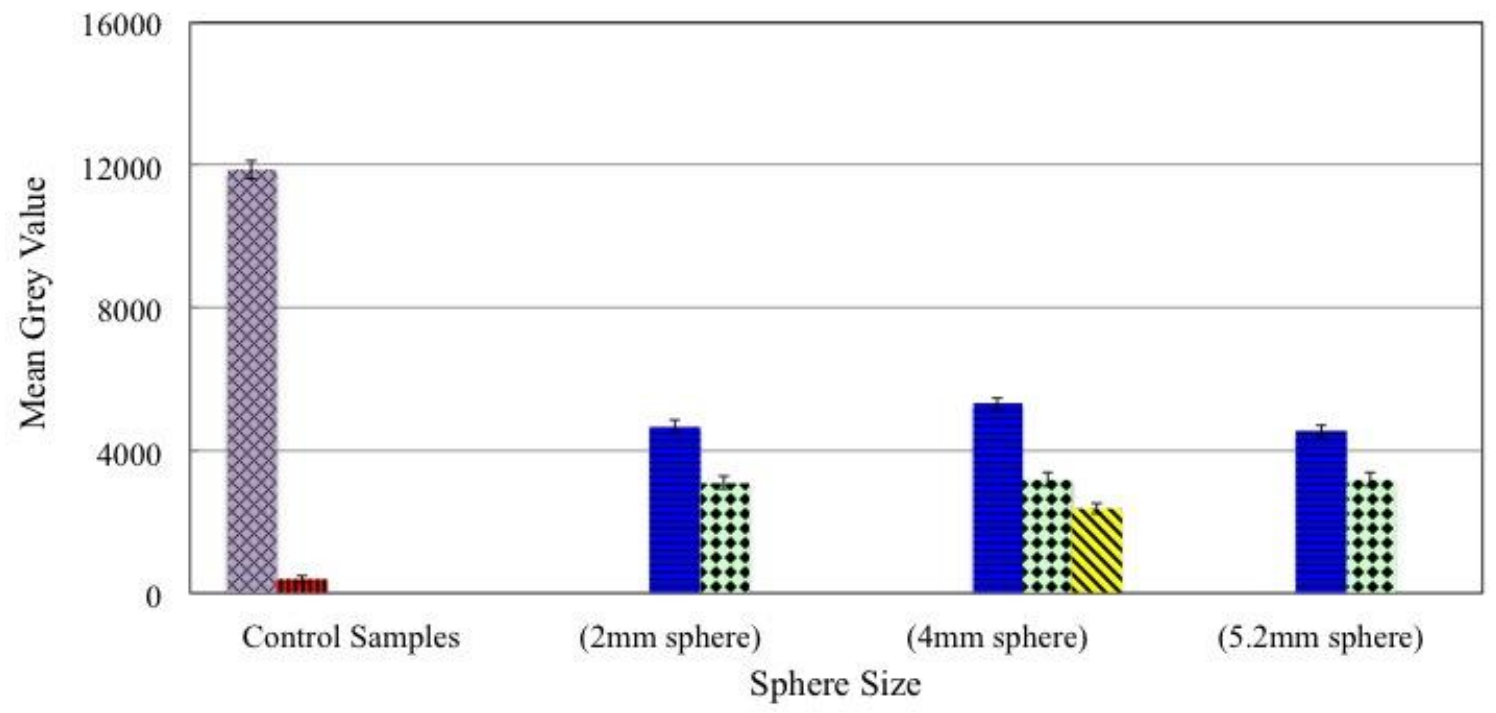

* Aluminum A356 mpure lead al-S CMF :?S-S CMF s 


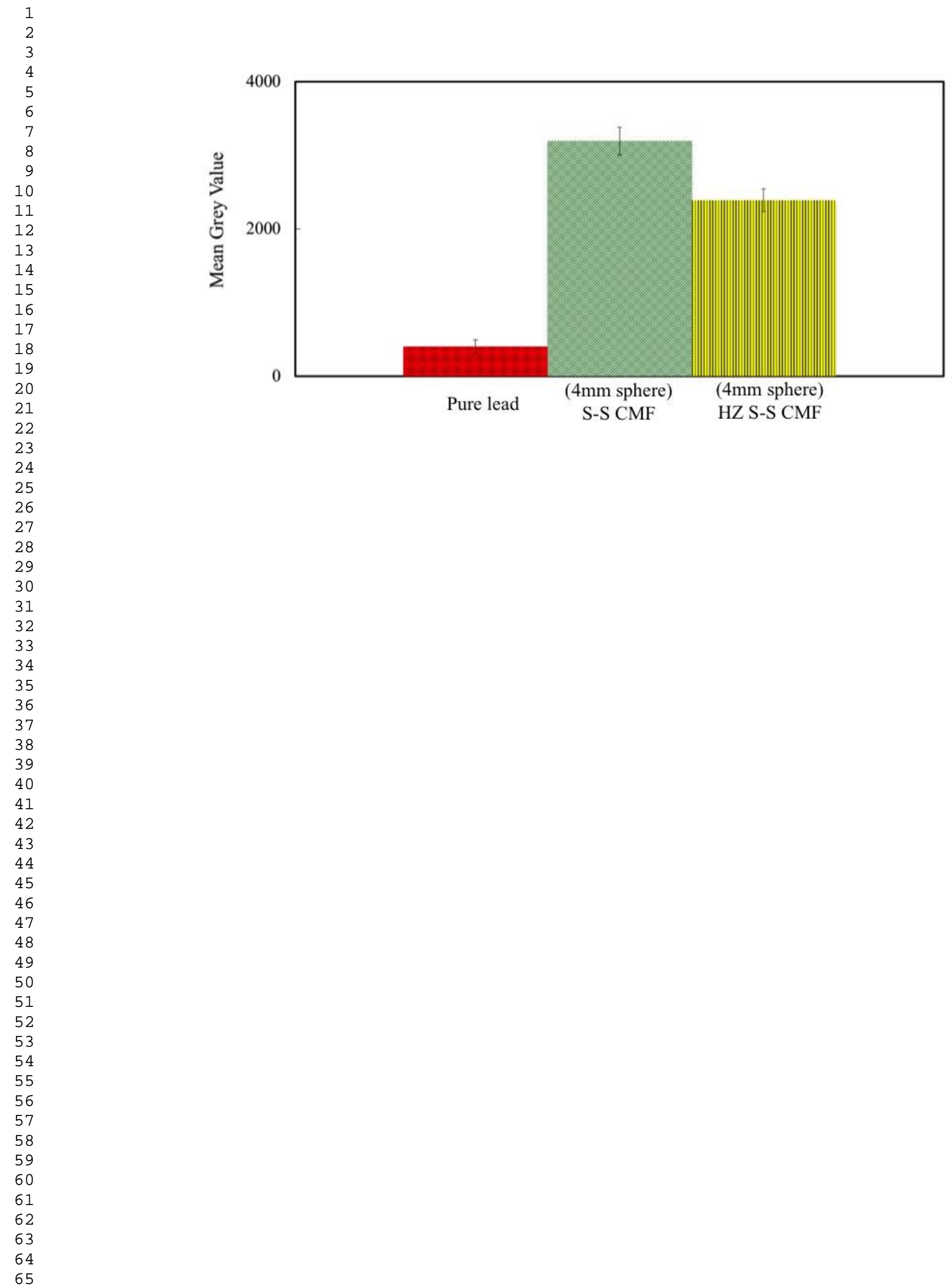



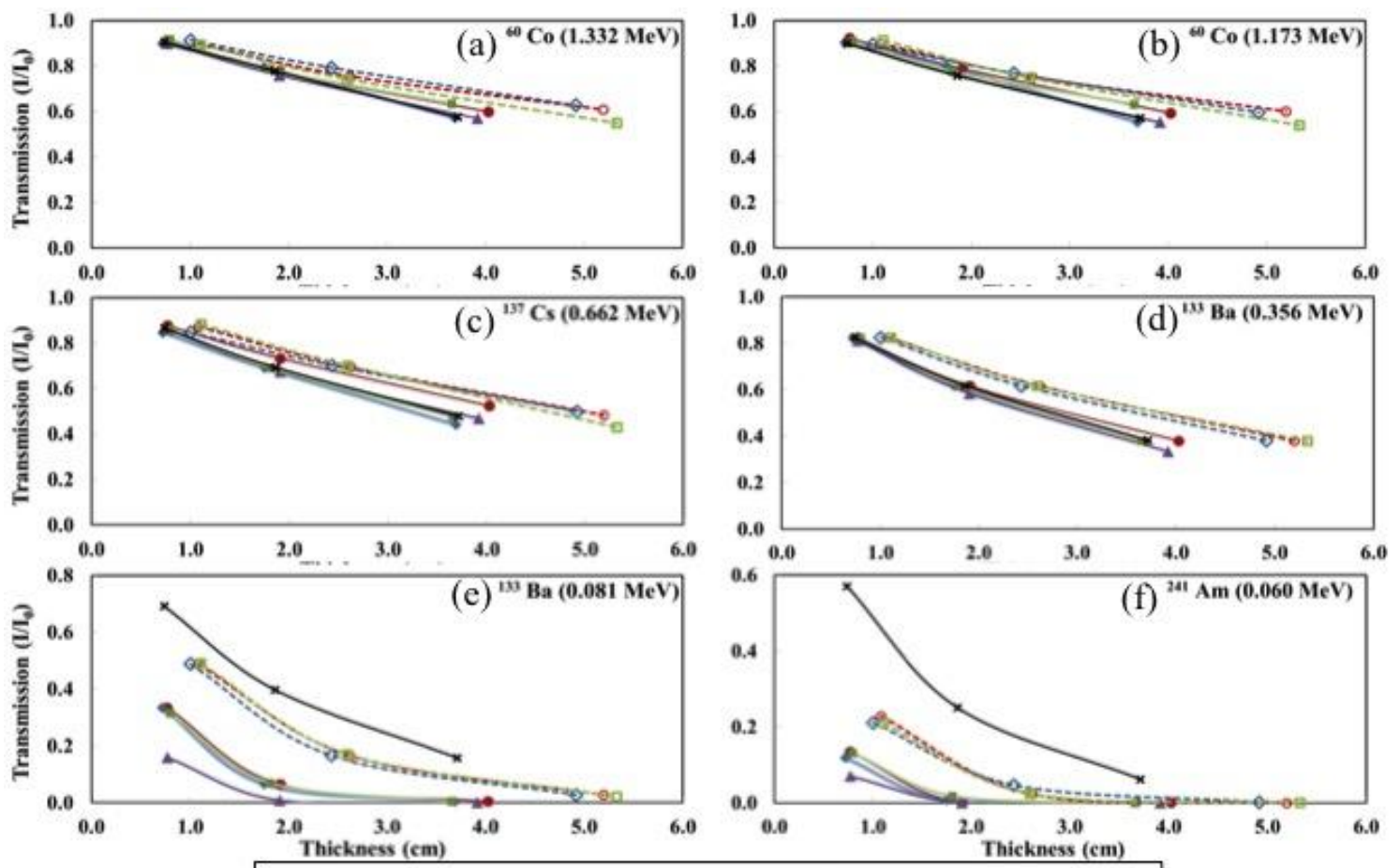

( $2 \mathrm{~mm}$ sphere) S-S CMF

(4mm sphere) S-S CMF

\#(4mm sphere) HZ S-S CMF

- $-(5.2 \mathrm{~mm}$ sphere) S-S CMF

$-\Theta-(2 \mathrm{~mm}$ sphere $) \mathrm{Al}-\mathrm{S}$ CMF $\diamond$-(4mm sphere) Al-S CMF

-75 (5.2 $\mathrm{mm}$ sphere) Al-S CMF 

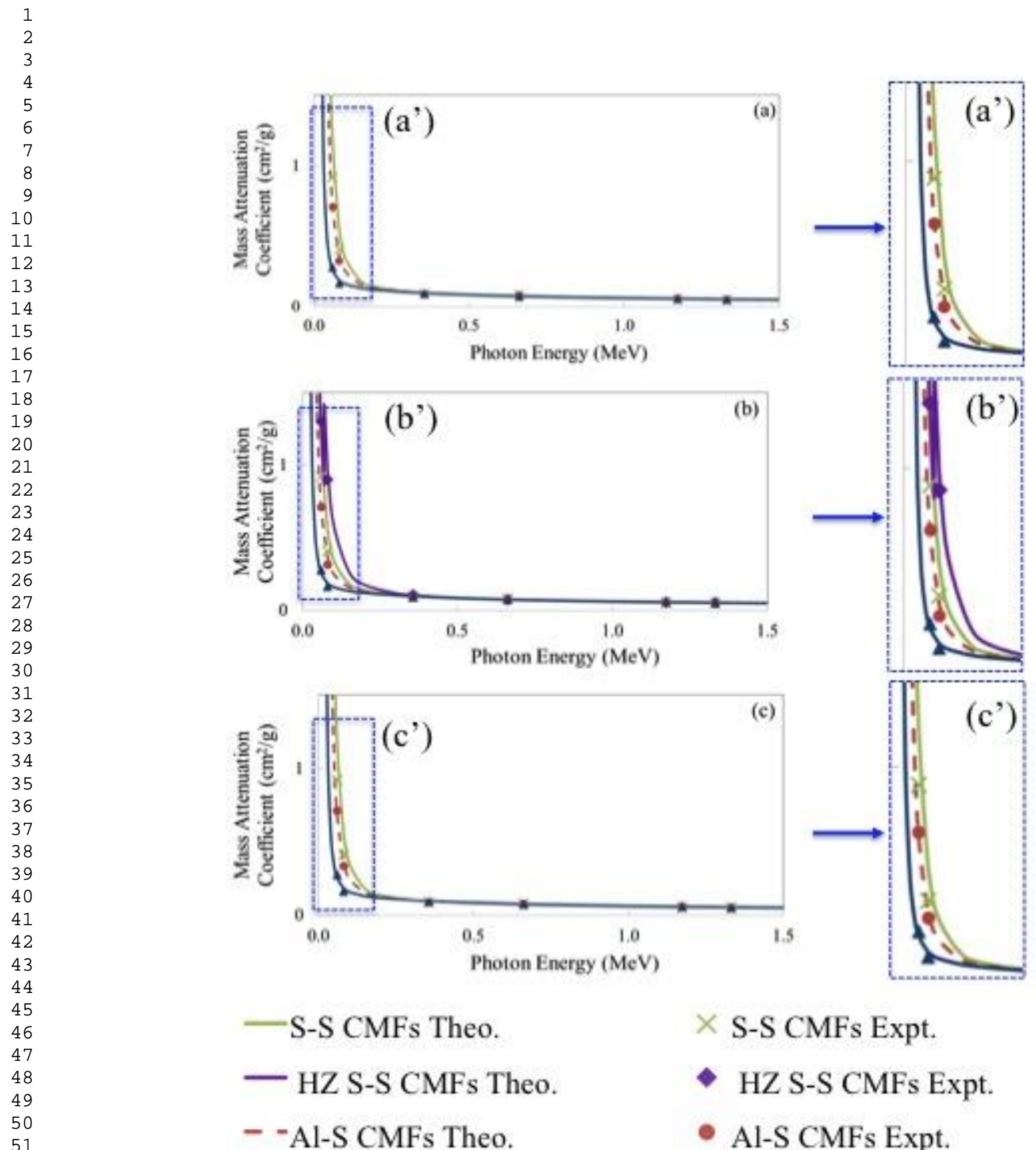

$\times$ S-S CMFs Expt. 




$=$ (2mm sphere) S-S CMF

‥ (4mm sphere) S-S CMF

- - (4mm sphere) HZ S-S CMF

$\rightarrow(5.2 \mathrm{~mm}$ sphere) S-S CMF

- $\cdot(2 \mathrm{~mm}$ sphere) Al-S CMF

$\Rightarrow$ (4mm sphere) Al-S CMF

$=(5.2 \mathrm{~mm}$ sphere $) \mathrm{Al}-\mathrm{S} \mathrm{CMF}$

-Aluminium A356 


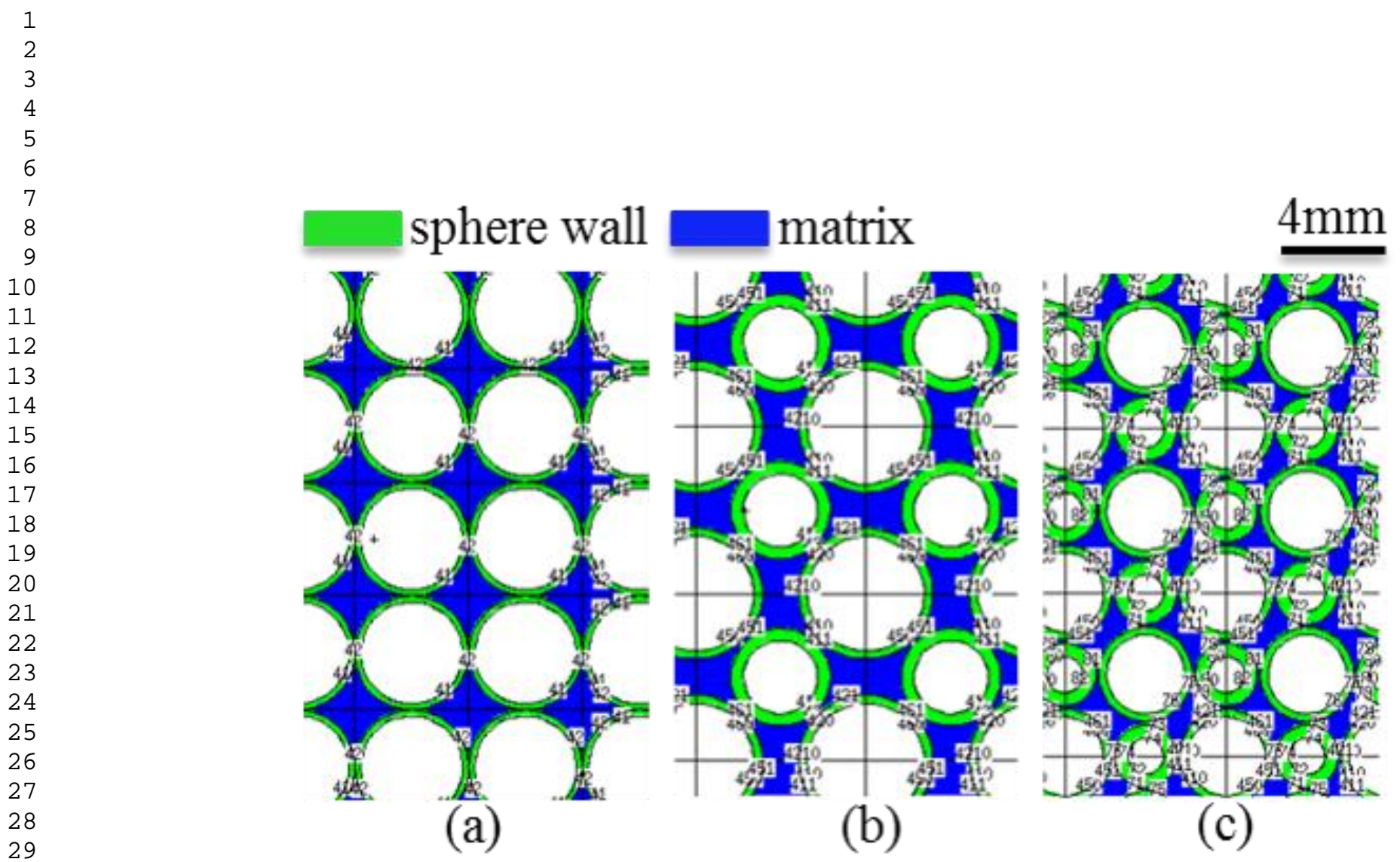




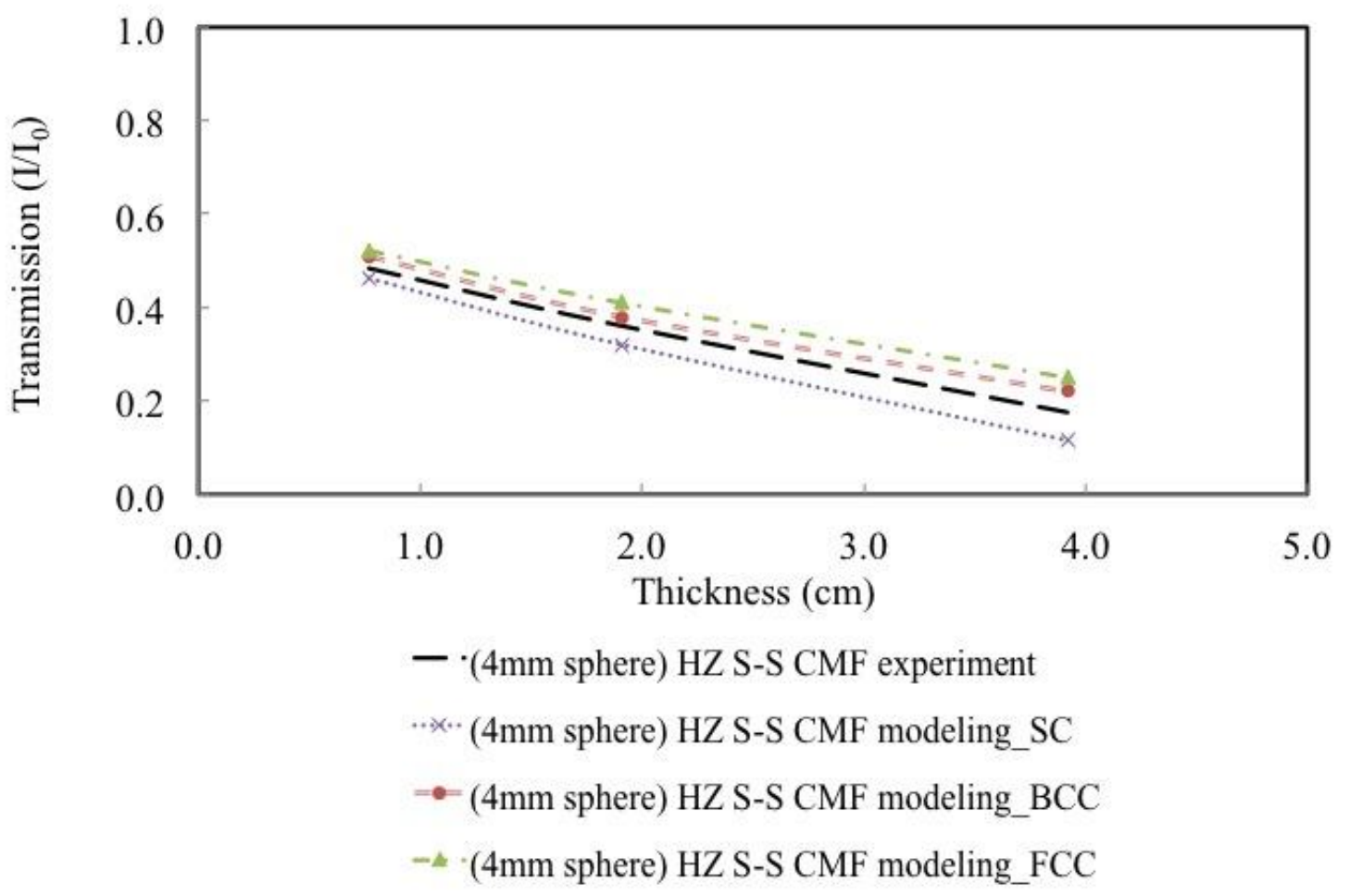




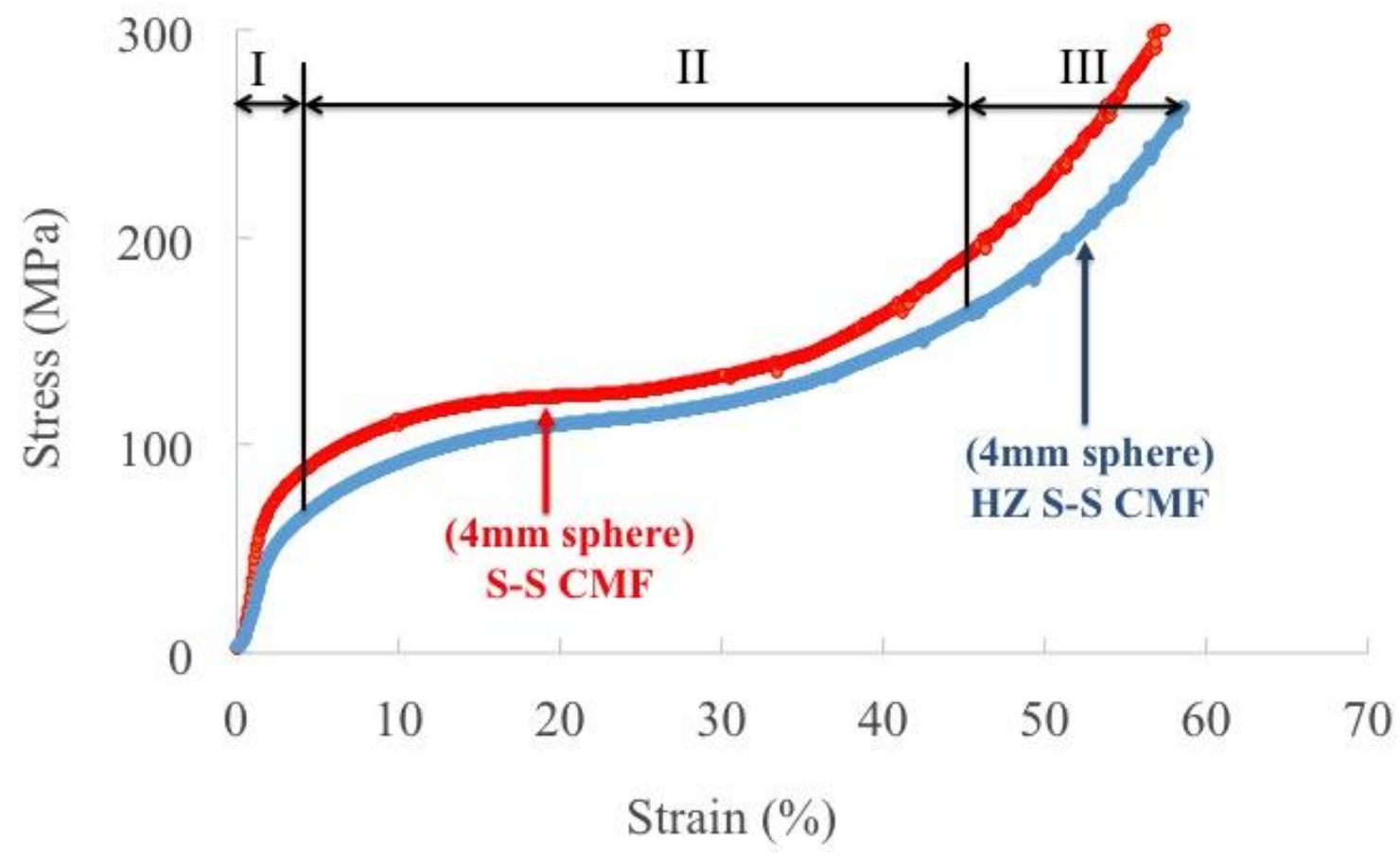




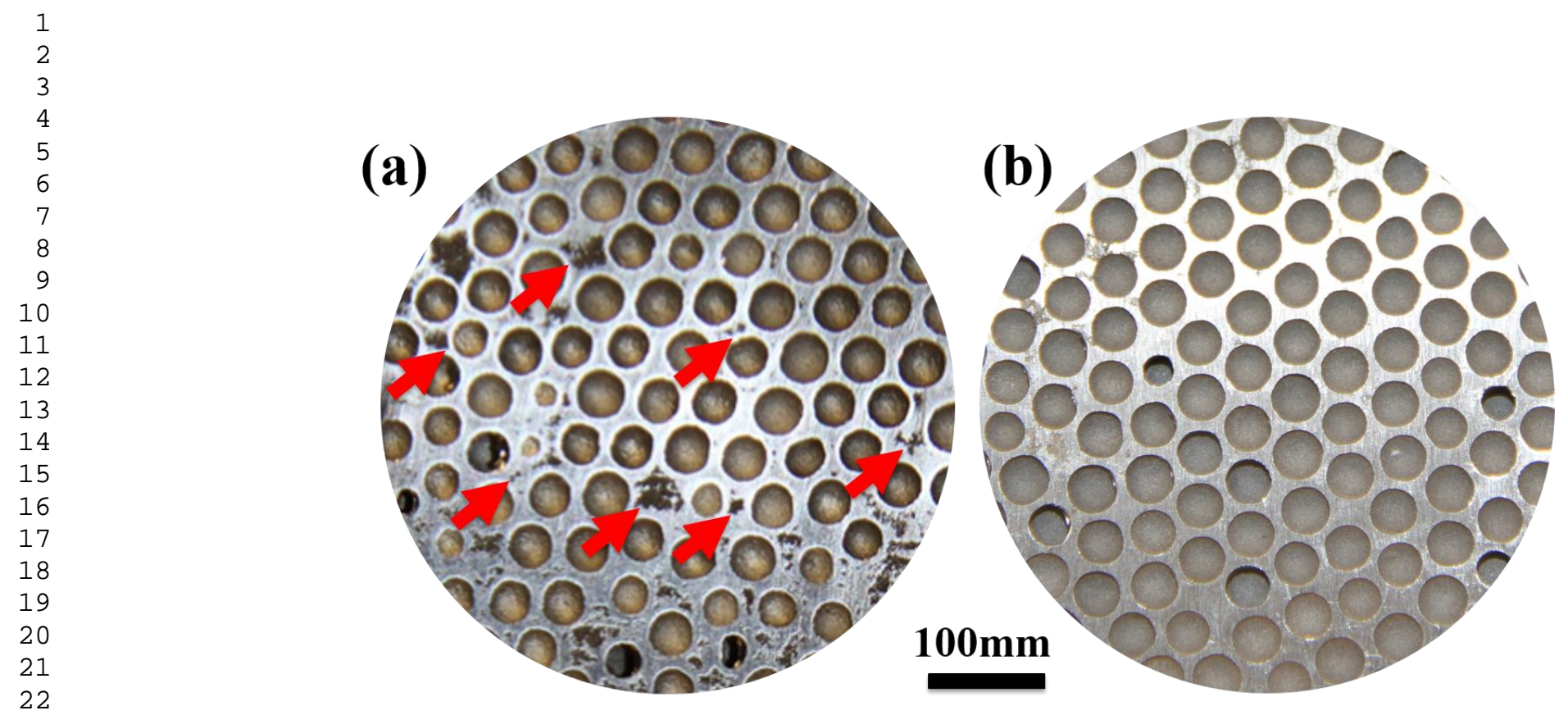


Table 1: Chemical compositions of different spheres in CMFs (wt\%)

\begin{tabular}{cccc}
\hline & $2 \mathrm{~mm}$ diameter & 4mm diameter & $5.2 \mathrm{~mm}$ diameter \\
\hline $\mathrm{C}$ & 0.68 & 0.58 & 0.87 \\
$\mathrm{Mn}$ & 0.13 & 0.15 & 0.07 \\
$\mathrm{Si}$ & 0.82 & 1.14 & 0.34 \\
$\mathrm{Cr}$ & 16.11 & 17.34 & 17.09 \\
$\mathrm{Ni}$ & 11.53 & 12.28 & 12.60 \\
$\mathrm{Mo}$ & 2.34 & 2.28 & 2.12 \\
$\mathrm{P}$ & -- & 0.009 & -- \\
$\mathrm{S}$ & -- & $<0.003$ & -- \\
$\mathrm{Cu}$ & -- & 0.04 & -- \\
$\mathrm{Co}$ & -- & 0.02 & -- \\
$\mathrm{Fe}$ & balance & balance & balance \\
\hline
\end{tabular}


Table 2: Chemical analysis and sieve analyses of matrix materials in CMFs (wt\%)

\begin{tabular}{|c|c|c|c|}
\hline Chemical analysis & 316L stainless steel & High-speed T15 steel & $\begin{array}{c}\text { Aluminum } \\
\text { A356.2 }\end{array}$ \\
\hline $\mathrm{C}$ & 0.03 & 1.60 & -- \\
\hline $\mathrm{Mn}$ & 2.00 & 0.40 & 0.28 \\
\hline $\mathrm{Si}$ & 1.00 & $\leq 0.40$ & 7.01 \\
\hline $\mathrm{Cr}$ & $16.00-18.00$ & 4.00 & 0.02 \\
\hline Co & -- & 5.00 & -- \\
\hline $\mathrm{Ni}$ & $10.00-14.00$ & -- & -- \\
\hline Mo & $2.00-3.00$ & $\leq 1.00$ & -- \\
\hline $\mathrm{P}$ & -- & -- & -- \\
\hline $\mathrm{S}$ & -- & 0.04 & -- \\
\hline $\mathrm{Cu}$ & -- & -- & 0.11 \\
\hline $\mathrm{Fe}$ & balance & balance & 0.50 \\
\hline $\mathrm{Mg}$ & -- & $\leq 0.40$ & 0.39 \\
\hline $\mathrm{Ti}$ & -- & -- & 0.09 \\
\hline $\mathrm{Zn}$ & -- & -- & 0.06 \\
\hline $\mathrm{Al}$ & -- & -- & balance \\
\hline $\mathrm{W}$ & -- & 12.50 & -- \\
\hline $\mathrm{V}$ & -- & 5.00 & -- \\
\hline \multicolumn{4}{|l|}{ Sieve analysis } \\
\hline Mesh & $\begin{array}{c}-325(95 \%) \\
-200 /+325(5 \%)\end{array}$ & $\begin{array}{c}-325(20 \%) \\
-100 /+325(78 \%) \\
+100(2 \%)\end{array}$ & -- \\
\hline Particle size & $\begin{array}{c}95 \%<44 \mu \mathrm{m} \\
44 \mu \mathrm{m}<5 \%<74 \mu \mathrm{m}\end{array}$ & $\begin{array}{c}20 \%<42 \mu \mathrm{m} \\
42 \mu \mathrm{m}<78 \%<149 \mu \mathrm{m} \\
2 \%<149 \mu \mathrm{m}\end{array}$ & -- \\
\hline Average particle size & $44.75 \mu \mathrm{m}$ & $86 \mu \mathrm{m}$ & -- \\
\hline
\end{tabular}




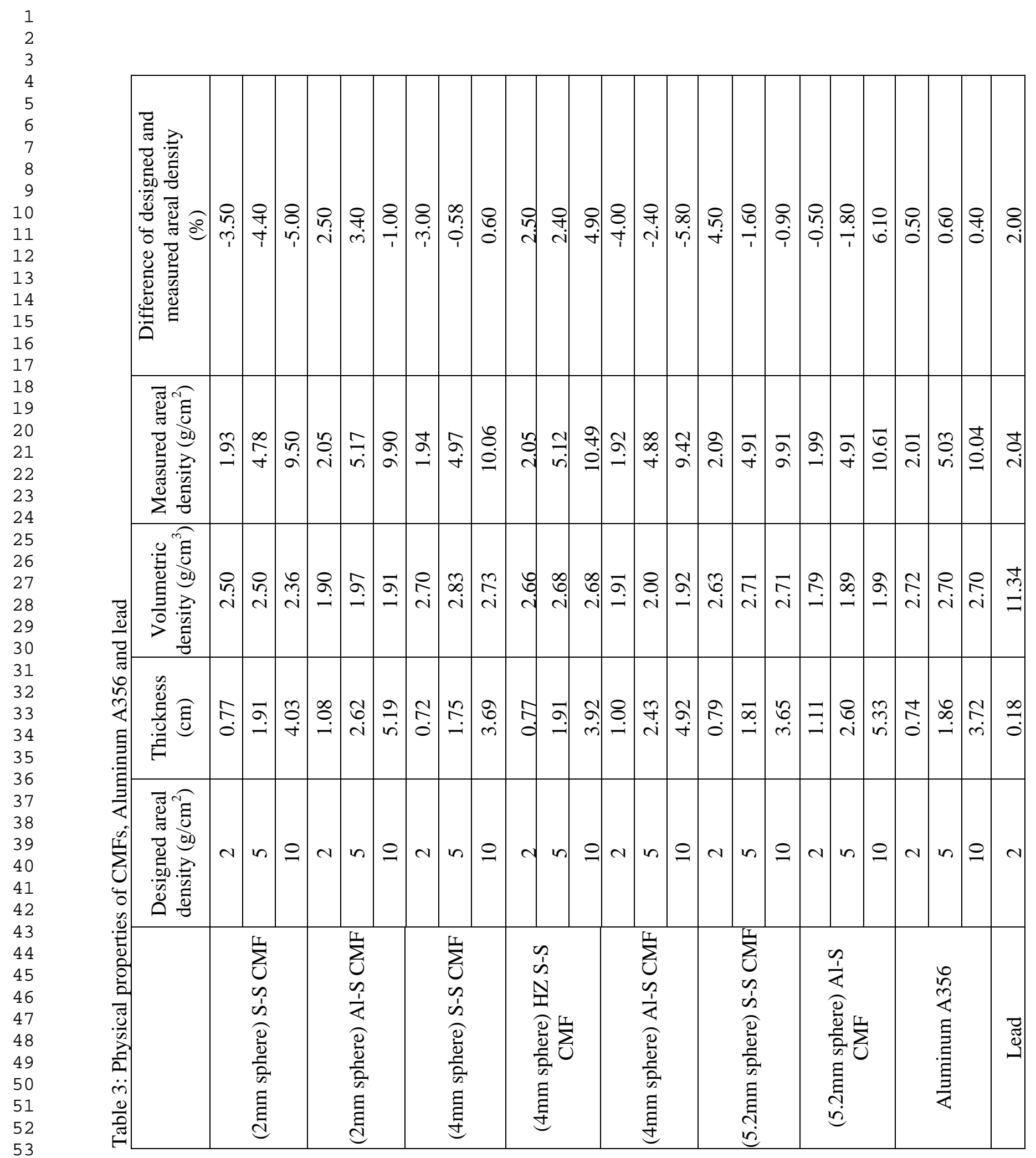


Table 4: Experimental and theoretical mass attenuation coefficients $\left(\mathrm{cm}^{2} / \mathrm{g}\right)$ for CMFs, Aluminum A356 and lead at X-ray energy of $100 \mathrm{kVp}$

\begin{tabular}{|c|c|c|}
\hline & Experimental & Theoretical \\
\hline (2mm sphere) S-S CMF & $0.4053 \pm 0.0059$ & 0.3477 \\
\hline (2mm sphere) Al-S CMF & $0.2089 \pm 0.0024$ & 0.2498 \\
\hline (4mm sphere) S-S CMF & $0.4044 \pm 0.0084$ & 0.3474 \\
\hline (4mm sphere) HZ S-S CMF & $0.9517 \pm 0.0090$ & 0.9416 \\
\hline (4mm sphere) Al-S CMF & $0.2081 \pm 0.0023$ & 0.2503 \\
\hline (5.2mm sphere) S-S CMF & $0.4044 \pm 0.0027$ & 0.3474 \\
\hline (5.2mm sphere) Al-S CMF & $0.2081 \pm 0.0025$ & 0.2499 \\
\hline Aluminum A356 & $0.1494 \pm 0.0062$ & 0.1572 \\
\hline Lead & $4.985 \pm 0.0016$ & 5.336 \\
\hline
\end{tabular}


Table 5: Effective atomic number of CMFs and Aluminum A356

\begin{tabular}{|c|c|c|}
\hline & Zeff $(0.081 \mathrm{MeV})$ & Zeff $(0.060 \mathrm{MeV})$ \\
\hline (2mm sphere) S-S CMF & 26.5 & 26.8 \\
\hline (2mm sphere) Al-S CMF & 22.5 & 20.9 \\
\hline (4mm sphere) S-S CMF & 26.5 & 26.8 \\
\hline (4mm sphere) HZ S-S CMF & 29.5 & 38.0 \\
\hline (4mm sphere) Al-S CMF & 22.4 & 21.4 \\
\hline (5.2mm sphere) S-S CMF & 26.5 & 26.7 \\
\hline (5.2mm sphere) Al-S CMF & 22.4 & 20.9 \\
\hline Aluminum A356 & 13.3 & 11.7 \\
\hline
\end{tabular}




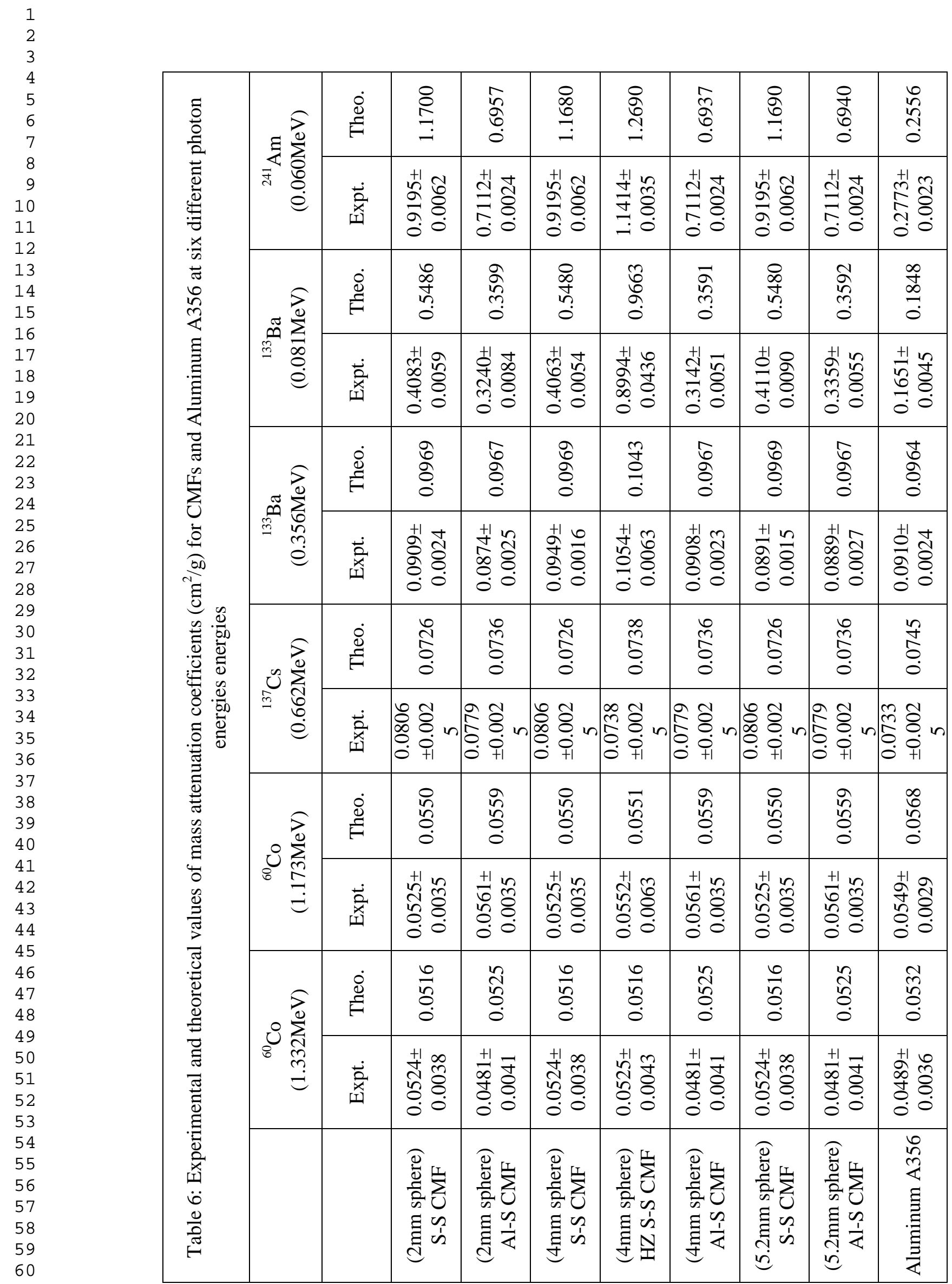

